\title{
Reciprocal classes of $p$-valently spirallike and $p$-valently Robertson functions
}

\author{
Neslihan Uyanik ${ }^{1}$, Hitoshi Shiraishi ${ }^{2}$, Shigeyoshi Owa ${ }^{2^{*}}$ and Yasar Polatoglu ${ }^{3}$
}

\footnotetext{
* Correspondence: shige21@ican. zaq.ne.jp

${ }^{2}$ Department of Mathematics, Kinki University, Higashi-Osaka, 577-8502 Osaka, Japan

Full list of author information is available at the end of the article
}

\section{Abstract \\ For $p$-valently spirallike and $p$-valently Robertson functions in the open unit disk $\mathbb{U}$, reciprocal classes $\mathcal{S}_{p}(\alpha, \beta)$, and $\mathcal{C}_{p}(\alpha, \beta)$ are introduced. The object of the present paper is to discuss some interesting properties for functions $f(z)$ belonging to the classes $\mathcal{S}_{p}(\alpha, \beta)$ and $\mathcal{C}_{p}(\alpha, \beta)$. \\ 2010 Mathematics Subject Classification \\ Primary 30C45}

Keywords: Reciprocal class, Subordination, Schwarz function, Robertson function, Miller and Mocanu lemma

\section{Introduction}

Let $\mathcal{A}_{p}$ be the class of functions $f(z)$ of the form

$$
f(z)=z^{p}+\sum_{n=p+1}^{\infty} a_{n} z^{n}
$$

which are analytic in the open unit disk $\mathbb{U}=\{z \in \mathbb{C}:|z|<1\}$.

For $f(z) \in \mathcal{A}_{p}$, we say that $f(z)$ belongs to the class $\mathcal{S}_{p}(\alpha, \beta)$ if it satisfies

$$
\operatorname{Re}\left(e^{i \alpha} \frac{z f^{\prime}(z)}{f(z)}\right)<\beta \quad(z \in \mathbb{U})
$$

for some real $\alpha\left(|\alpha|<\frac{\pi}{2}\right)$ and $\beta(\beta>p \cos \alpha)$.

When $\alpha=0$, the class $\mathcal{S}_{p}(0, \beta)$ was studied by Polatoglu et al. [1], and the classes $\mathcal{S}_{1}(0, \beta)$ and $\mathcal{C}_{1}(0, \beta)$ were introduced by Owa and Nishiwaki [2].

Further, let $\mathcal{C}_{p}(\alpha, \beta)$ denote the subclass of $\mathcal{A}_{p}$ consisting of functions $f(z)$, which satisfy

$$
\operatorname{Re}\left\{e^{i \alpha}\left(1+\frac{z f^{\prime \prime}(z)}{f^{\prime}(z)}\right)\right\}<\beta \quad(z \in \mathbb{U})
$$

for some real $\alpha\left(|\alpha|<\frac{\pi}{2}\right)$ and $\beta(\beta>p \cos \alpha)$.

We note that $f(z) \in \mathcal{C}_{p}(\alpha, \beta)$ if and only if $\frac{z f^{\prime}(z)}{p} \in \mathcal{S}_{p}(\alpha, \beta)$, and that, $f(z) \in \mathcal{S}_{p}(\alpha, \beta)$ if and only if $p \int_{0}^{z} \frac{f(t)}{t} \mathrm{~d} t \in \mathcal{C}_{p}(\alpha, \beta)$.

\section{Springer}

(C) 2011 Uyanik et al; licensee Springer. This is an Open Access article distributed under the terms of the Creative Commons Attribution License (http://creativecommons.org/licenses/by/2.0), which permits unrestricted use, distribution, and reproduction in any medium, provided the original work is properly cited. 
Remark 1 If $f(z) \in \mathcal{A}_{p}$ satisfies

$$
\operatorname{Re}\left(e^{i \alpha} \frac{z f^{\prime}(z)}{f(z)}\right)>0 \quad(z \in \mathbb{U}),
$$

then we say that $f(z)$ is $p$-valently spirallike in $\mathbb{U}\left(\mathrm{cf}\right.$. [1]). Also, if $f(z) \in \mathcal{A}_{p}$ satisfies

$$
\operatorname{Re}\left\{e^{i \alpha}\left(1+\frac{z f^{\prime \prime}(z)}{f^{\prime}(z)}\right)\right\}>0 \quad(z \in \mathbb{U})
$$

then $f(z)$ is said to be $p$-valently Robertson function in $\mathbb{U}$ (cf. $[3,4])$. Therefore, $\mathcal{S}_{p}(\alpha, \beta)$ defined by (1.2) is the reciprocal class of $p$-valently spirallike functions in $\mathbb{U}$, and $\mathcal{C}_{p}(\alpha, \beta)$ defined by (1.3) is the reciprocal class of $p$-valently Robertson functions in $\mathbb{U}$.

Let $\mathcal{P}$ be the class of functions $p(z)$ of the form

$$
p(z)=1+\sum_{n=1}^{\infty} c_{n} z^{n} \quad(z \in \mathbb{U})
$$

that are analytic in $\mathbb{U}$ and satisfy $\operatorname{Re} p(z)>0(z \in \mathbb{U})$. A function $p(z) \in \mathcal{P}$ is called the Carathéodory function and satisfies

$$
\left|c_{n}\right| \leqq 2 \quad(n=1,2,3, \ldots)
$$

with the equality for $p(z)=\frac{1+z}{1-z}$ (cf. [5]).

For analytic functions $g(z)$ and $h(z)$ in $\mathbb{U}$, we say that $g(z)$ is subordinate to $h(z)$ if there exists an analytic function $w(z)$ in $\mathbb{U}$ with $w(0)=0$ and $|w(z)|<1(z \in \mathbb{U})$, and such that $g(z)=h(w(z))$. We denote this subordination by

$$
g(z) \prec h(z) \quad(z \in \mathbb{U}) .
$$

If $h(z)$ is univalent in $\mathbb{U}$, then this subordination (1.6) is equivalent to $g(0)=h(0)$ and $g(\mathbb{U}) \subset h(\mathbb{U})$ (cf. [5]).

\section{Subordinations for classes}

We consider subordination properties of function $f(z)$ in the classes $\mathcal{S}_{p}(\alpha, \beta)$ and $\mathcal{C}_{p}(\alpha, \beta)$

Theorem 1 A function $f(z)$ belongs to the class $\mathcal{S}_{p}(\alpha, \beta)$ if and only if

$$
e^{i \alpha} \frac{z f^{\prime}(z)}{f(z)} \prec 2 \beta-p e^{-i \alpha}+\frac{2(p \cos \alpha-\beta)}{1-z} \quad(z \in \mathbb{U})
$$

for some real $\alpha\left(|\alpha|<\frac{\pi}{2}\right)$ and $\beta(\beta>p \cos \alpha)$.

The result is sharp for $f(z)$ given by

$$
f(z)=\frac{z^{p}}{(1-z)^{2 e^{-i \alpha}(p \cos \alpha-\beta)}} .
$$

Proof. Let $f(z) \in \mathcal{S}_{p}(\alpha, \beta)$. If we define the function $w(z)$ by

$$
\frac{\beta-\mathrm{e}^{i \alpha} \frac{z f^{\prime}(z)}{f(z)}+i p \sin \alpha}{\beta-p \cos \alpha}=\frac{1+w(z)}{1-w(z)} \quad(w(z) \neq 1),
$$


then we know that $w(z)$ is analytic in $\mathbb{U}, w(0)=0$, and

$$
\operatorname{Re}\left(\frac{1+w(z)}{1-w(z)}\right)>0 \quad(z \in \mathbb{U}) .
$$

Therefore, we have that $|w(z)|<1(z \in \mathbb{U})$. If follows from (2.3) that

$$
\mathrm{e}^{i \alpha} \frac{z f^{\prime}(z)}{f(z)}=2 \beta-p \mathrm{e}^{-i \alpha}+\frac{2(p \cos \alpha-\beta)}{1-w(z)} \quad(z \in \mathbb{U}),
$$

which is equivalent to the subordination (2.1).

Conversely, we suppose that the subordination (2.1) holds true. Then, we have that

$$
e^{i \alpha} \frac{z f^{\prime}(z)}{f(z)}=2 \beta-p e^{-i \alpha}+\frac{2(p \cos \alpha-\beta)}{1-w(z)} \quad(z \in \mathbb{U}),
$$

for some Shwarz function $w(z)$, which is analytic in $\mathbb{U}, w(0)=0$, and $|w(z)|<1(z \in \mathbb{U})$. It is easy to see that the equality (2.6) is equivalent to the equality (2.3). Since

$$
\operatorname{Re}\left(\frac{1+w(z)}{1-w(z)}\right)=\operatorname{Re}\left\{\frac{\beta-\mathrm{e}^{i \alpha} \frac{z f^{\prime}(z)}{f(z)}+i p \sin \alpha}{\beta-p \cos \alpha}\right\}>0 \quad(z \in \mathbb{U})
$$

we conclude that

$$
\operatorname{Re}\left(\beta-\mathrm{e}^{i \alpha} \frac{z f^{\prime}(z)}{f(z)}\right)>0 \quad(z \in \mathbb{U}),
$$

which shows that $f(z) \in \mathcal{S}_{p}(\alpha, \beta)$.

Finally, we consider the function $f(z)$ given by (2.2). Then, $f(z)$ satisfies

$$
\mathrm{e}^{i \alpha} \frac{z f^{\prime}(z)}{f(z)}=2 \beta-p \mathrm{e}^{-i \alpha}+\frac{2(p \cos \alpha-\beta)}{1-z} .
$$

This completes the proof of the theorem.

Noting that $f(z) \in \mathcal{C}_{p}(\alpha, \beta)$ if and only if $\frac{z f^{\prime}(z)}{p} \in \mathcal{S}_{p}(\alpha, \beta)$, we also have

Corollary 1 A function $f(z)$ belongs to the class $\mathcal{C}_{p}(\alpha, \beta)$ if and only if

$$
e^{i \alpha}\left(1+\frac{z f^{\prime \prime}(z)}{f^{\prime}(z)}\right) \prec 2 \beta-p e^{-i \alpha}+\frac{2(p \cos \alpha-\beta)}{1-z} \quad(z \in \mathbb{U})
$$

for some real $\alpha\left(|\alpha|<\frac{\pi}{2}\right)$ and $\beta(\beta>p \cos \alpha)$.

The result is sharp for $f(z)$ given by

$$
f^{\prime}(z)=\frac{p z^{p-1}}{(1-z)^{2 e^{-i \alpha}(p \cos \alpha-\beta)}} .
$$

\section{Coefficient inequalities}

Applying the properties for Carathéodory functions, we discuss the coefficient inequalities for $f(z)$ in the classes $\mathcal{S}_{p}(\alpha, \beta)$ and $\mathcal{C}_{p}(\alpha, \beta)$. 
Theorem 2 If $f(z)$ belongs to the class $\mathcal{S}_{p}(\alpha, \beta)$, then

$$
\left|a_{p+k}\right| \leqq \frac{1}{k !} \prod_{j=0}^{k-1}(2(\beta-p \cos \alpha)+j) \quad(k=1,2,3, \ldots)
$$

The result is sharp for

$$
f(z)=\frac{z^{p}}{(1-z)^{2(p-\beta)}}
$$

for $\alpha=0$.

Proof. In view of Theorem 1, we can consider the function $w(z)$ given by (2.3) for $f(z) \in \mathcal{S}_{p}(\alpha, \beta)$. Since $w(z)$ is the Schwarz function, the function $q(z)$ defined by

$$
q(z)=\frac{\beta-e^{i \alpha} \frac{z f^{\prime}(z)}{f(z)}+i p \sin \alpha}{\beta-p \cos \alpha}
$$

is the Carathéodory function. If we write that

$$
q(z)=1+\sum_{n=1}^{\infty} c_{n} z^{n}
$$

then we see that

$$
\left|c_{n}\right| \leqq 2 \quad(n=1,2,3, \ldots)
$$

and the equality holds true for $q(z)=\frac{1+z}{1-z}$ and its rotation. It is to be noted that the equation (3.3) is equivalent to

$$
\mathrm{e}^{i \alpha} \frac{z f^{\prime}(z)}{f(z)}=\beta+i p \sin \alpha-(\beta-p \cos \alpha) q(z)
$$

This gives us that

$$
\begin{aligned}
& e^{i \alpha}\left(p z^{p}+\sum_{n=p+1}^{\infty} n a_{n} z^{n}\right) \\
& =\left\{p \mathrm{e}^{i \alpha}-(\beta-p \cos \alpha)\left(\sum_{n=1}^{\infty} c_{n} z^{n}\right)\right\}\left(z^{p}+\sum_{n=p+1}^{\infty} a_{n} z^{n}\right),
\end{aligned}
$$

which implies that

$$
\mathrm{e}^{i \alpha}(n-p) a_{n}=-(\beta-p \cos \alpha)\left(c_{n-1}+a_{2} c_{n-2}+\cdots+a_{n-1} c_{1}\right) .
$$

It follows from (3.7) that

$$
\left|a_{n}\right| \leqq \frac{2(\beta-p \cos \alpha)}{n-p}\left(1+\left|a_{2}\right|+\left|a_{3}\right|+\cdots+\left|a_{n-1}\right|\right) .
$$

If $n=p+1$, then we have that

$$
\left|a_{p+1}\right| \leqq 2(\beta-p \cos \alpha) \text {. }
$$


If $n=p+2$, then we also have that

$$
\begin{aligned}
& \left|a_{p+2}\right| \leqq \frac{2(\beta-p \cos \alpha)}{2}\left(1+\left|a_{2}\right|\right) \\
& \leqq(\beta-p \cos \alpha)(1+2(\beta-p \cos \alpha)) .
\end{aligned}
$$

Thus, the coefficient inequality (3.1) is true for $n=p+1$ and $n=p+2$. Next, we suppose that (3.1) holds true for $n=p+1, p+2, p+3, \ldots, p+k-1$. Then

$$
\begin{gathered}
\left|a_{p+k}\right| \leqq \frac{2(\beta-p \cos \alpha)}{k}\left(1+\left|a_{2}\right|+\left|a_{3}\right|+\cdots+\left|a_{p+k-1}\right|\right) \\
\leqq \frac{2(\beta-p \cos \alpha)}{k}\left\{1+2(\beta-p \cos \alpha)+\frac{2(\beta-p \cos \alpha)}{2}(1+2(\beta-p \cos \alpha))\right. \\
+\frac{2(\beta-p \cos \alpha)}{3}(1+2(\beta-p \cos \alpha))\left(1+\frac{2(\beta-p \cos \alpha)}{2}\right) \\
\left.+\cdots+\frac{1}{(k-1) !} \prod_{j=0}^{k-2}(2(\beta-p \cos )+j)\right\} \\
=\frac{2(\beta-p \cos \alpha)}{k}(1+2(\beta-p \cos \alpha))\left\{1+\frac{2(\beta-p \cos \alpha)}{2}\right. \\
+\left(1+\frac{2(\beta-p \cos \alpha)}{2}\right) \frac{2(\beta-p \cos \alpha)}{3} \\
\left.+\cdots+\frac{2(\beta-p \cos \alpha)}{(k-1) !} \prod_{j=1}^{k-2}(2(\beta-p \cos \alpha)+j)\right\} \\
=\frac{1}{k !} \prod_{j=0}^{k-1}(2(\beta-p \cos \alpha)+j) .
\end{gathered}
$$

This means that the inequality (3.1) holds true for $n=p+k$. Therefore, by the mathematical induction, we prove the coefficient inequality (3.1).

Finally, let us consider the function $f(z)$ given by (3.2). Then, $f(z)$ can be written by

$$
\begin{gathered}
f(z)=z^{p}\left(\sum_{j=0}^{\infty}\left(\begin{array}{c}
2(\beta-p) \\
j
\end{array}\right)(-z)^{j}\right) \\
=z^{p}+2(\beta-p) z^{p+1}+\cdots+\left(\frac{1}{k !} \prod_{j=0}^{k-1}(2(\beta-p)+j) z^{p+k}\right)+\ldots
\end{gathered}
$$

Thus, this function $f(z)$ satisfies the equality in (3.1). $\quad \square$

Corollary 2 If $f(z)$ belongs to the class $\mathcal{C}_{p}(\alpha, \beta)$, then

$$
\left|a_{p+k}\right| \leqq \frac{1}{(k-1) !} \prod_{j=0}^{k-1}(2(\beta-p \cos \alpha)+j) \quad(k=1,2,3, \ldots)
$$

The result is sharp for $f(z)$ defined by

$$
f^{\prime}(z)=\frac{p z^{p-1}}{(1-z)^{2(p-\beta)}}
$$

for $\alpha=0$. 
Remark 2 We know that the extremal functions for $f(z) \in \mathcal{S}_{p}(\alpha, \beta)$ is $f(z)$ given by (2.2) and for $f(z) \in \mathcal{C}_{p}(\alpha, \beta)$ is $f(z)$ given by (2.11). But, we see that

$$
\left|a_{p+k}\right| \leqq \frac{1}{k !} \prod_{j=0}^{k-1}\left|2 e^{-i \alpha}(\beta-p \cos \alpha)+j\right|
$$

and

$$
\left|a_{p+k}\right| \leqq \frac{1}{(k-1) !} \prod_{j=0}^{k-1}\left|2 e^{-i \alpha}(\beta-p \cos \alpha)+j\right|
$$

for such functions.

Therefore, the extremal functions for $f(z) \in \mathcal{S}_{p}(\alpha, \beta)$ and $f(z) \in \mathcal{C}_{p}(\alpha, \beta)$ do not satisfy the equalities in (3.1) and (3.13), respectively.

Furthermore, if we consider $\alpha=0$ in Theorem 2, then we obtain the corresponding result due to Polatoglu et al. [1].

\section{Inequalities for the real parts}

We discuss some problems of inequalities for the real parts of $\frac{z f^{\prime}(z)}{f(z)}$.

Theorem 3 If $f(z) \in \mathcal{S}_{p}(\alpha, \beta)$, then we have

$$
\frac{p \cos \alpha-(2 \beta-p \cos \alpha) r}{1-r} \leqq \operatorname{Re}\left(e^{i \alpha} \frac{z f^{\prime}(z)}{f(z)}\right) \leqq \frac{p \cos \alpha+(2 \beta-p \cos \alpha) r}{1+r}
$$

for $|z|=r<1$. The equalities hold true for $f(z)$ given by (2.2).

Proof. By virtue of Theorem 1, we consider the function $g(z)$ defined by

$$
g(z)=2 \beta-p e^{-i \alpha}+\frac{2(p \cos \alpha-\beta)}{1-z} \quad(z \in \mathbb{U}) .
$$

Letting $z=r \mathrm{e}^{i \theta}(0 \leqq r<1)$, we see that

$$
\operatorname{Reg}(z)=2 \beta-p \cos \alpha+\frac{2(p \cos \alpha-\beta)(1-r \cos \theta)}{1+r^{2}-2 r \cos \theta} .
$$

Let us define

$$
h(t)=\frac{1-r t}{1+r^{2}-2 r t} \quad(t=\cos \theta) .
$$

Then, we know that $h^{\prime}(t) \geqq 0$. This implies that

$$
2 \beta-p \cos \alpha+\frac{2(p \cos \alpha-\beta)}{1-r} \leqq \operatorname{Reg}(z) \leqq 2 \beta-p \cos \alpha+\frac{2(p \cos \alpha-\beta)}{1+r},
$$

which is equivalent to

$$
\frac{p \cos \alpha-(2 \beta-p \cos \alpha) r}{1-r} \leqq \operatorname{Reg}(z) \leqq \frac{p \cos \alpha+(2 \beta-p \cos \alpha) r}{1+r} .
$$

Noting that $\mathrm{e}^{i \alpha} \frac{z f^{\prime}(z)}{f(z)} \prec g(z)(z \in \mathbb{U})$ by Theorem 1 and $g(z)$ is univalent in $\mathbb{U}$, we prove the inequality (4.1). Since the subordination (2.1) is sharp for $f(z)$ given by (2.2), we say that the equalities in (4.1) are attained by the function $f(z)$ given by (2.2). 
Taking $\alpha=0$ in Theorem 3, we have

Corollary 3 If $f(z) \in \mathcal{S}_{p}(0, \beta)$, then

$$
\frac{p-(2 \beta-p) r}{1-r} \leqq \operatorname{Re}\left(\frac{z f^{\prime}(z)}{f(z)}\right) \leqq \frac{p+(2 \beta-p) r}{1+r}
$$

for $|z|=r<1$. The equalities hold true for

$$
f(z)=\frac{z^{p}}{(1-z)^{2(p-\beta)}} .
$$

Corollary 4 If $f(z) \in \mathcal{C}_{p}(\alpha, \beta)$, then we have

$$
\frac{p \cos \alpha-(2 \beta-p \cos \alpha) r}{1-r} \leqq \operatorname{Re}\left\{e^{i \alpha}\left(1+\frac{z f^{\prime \prime}(z)}{f^{\prime}(z)}\right)\right\} \leqq \frac{p \cos \alpha+(2 \beta-p \cos \alpha) r}{1+r}
$$

for $|z|=r<1$. The equalities hold true for $f(z)$ defined by (2.11).

Corollary 5 If $f(z) \in \mathcal{C}_{p}(0, \beta)$, then

$$
\frac{p-(2 \beta-p) r}{1-r} \leqq \operatorname{Re}\left(1+\frac{z f^{\prime \prime}(z)}{f^{\prime}(z)}\right) \leqq \frac{p+(2 \beta-p) r}{1+r}
$$

for $|z|=r<1$. The equalities hold true for $f(z)$ defined by

$$
f^{\prime}(z)=\frac{p z^{p-1}}{(1-z)^{2(p-\beta)}}
$$

\section{Sufficient conditions}

We consider some sufficient conditions for $f(z)$ to be in the classes $\mathcal{S}_{p}(0, \beta)$ and $\mathcal{C}_{p}(0, \beta)$.

To discuss our sufficient conditions, we have to recall here the following lemma by Miller and Mocanu [6] (also due to Jack [7]).

Lemma 1 Let $w(z)$ be analytic in $\mathbb{U}$ with $w(0)=0$. If there exists a point $z_{0} \in \mathbb{U}$ such that

$$
\max _{|z| \leqq\left|z_{0}\right|}|w(z)|=\left|w\left(z_{0}\right)\right|
$$

then we can write

$$
z_{0} w^{\prime}\left(z_{0}\right)=k w\left(z_{0}\right)
$$

where $k$ is real and $k \geqq 1$.

Applying Lemma 1, we derive

Theorem 4 If $f(z) \in \mathcal{A}_{p}$ satisfies

$$
\operatorname{Re}\left(\frac{z f^{\prime}(z)}{f(z)}-\frac{z f^{\prime \prime}(z)}{f^{\prime}(z)}\right)>\frac{p+\beta}{2 \beta} \quad(z \in \mathbb{U})
$$

for some real $\beta>p$, then $f(z) \in \mathcal{S}_{p}(0, \beta)$.

Proof. Let us define the function $w(z)$ by

$$
\frac{z f^{\prime}(z)}{f(z)}=\frac{p+(p-2 \beta) w(z)}{1-w(z)} \quad(w(z) \neq 1) .
$$


Then we see that $w(z)$ is analytic in $\mathbb{U}$ and $w(0)=0$.

It follows from (5.4) that

$$
\begin{aligned}
\operatorname{Re}\left(\frac{z f^{\prime}(z)}{f(z)}-\frac{z f^{\prime \prime}(z)}{f^{\prime}(z)}\right) & =\operatorname{Re}\left(1-\frac{(p-2 \beta) z w^{\prime}(z)}{p+(p-2 \beta) w(z)}-\frac{z w^{\prime}(z)}{1-w(z)}\right) \\
& >\frac{p+\beta}{2 \beta} \quad(z \in \mathbb{U}) .
\end{aligned}
$$

We suppose that there exists a point $z_{0} \in \mathbb{U}$ such that

$$
\max _{|z| \leqq\left|z_{0}\right|}|w(z)|=\left|w\left(z_{0}\right)\right|=1 .
$$

Then, Lemma 1 gives us that $w\left(z_{0}\right)=\mathrm{e}^{i \theta}$ and $z_{0} w^{\prime}\left(z_{0}\right)=k \mathrm{e}^{i \theta}$. For such a point $z_{0}$, we have that

$$
\begin{gathered}
\operatorname{Re}\left(\frac{z_{0} f^{\prime}\left(z_{0}\right)}{f\left(z_{0}\right)}-\frac{z_{0} f^{\prime \prime}\left(z_{0}\right)}{f^{\prime}\left(z_{0}\right)}\right)=\operatorname{Re}\left(1-\frac{(p-2 \beta) k e^{i \theta}}{p+(p-2 \beta) e^{i \theta}}-\frac{k e^{i \theta}}{1-e^{i \theta}}\right) \\
=1+\frac{(2 \beta-p) k(p \cos \theta+p-2 \beta)}{p^{2}+(p-2 \beta)^{2}+2 p(p-2 \beta) \cos \theta}+\frac{k}{2} \\
\leqq 1-\frac{(2 \beta-p) k}{2 \beta}+\frac{k}{2} \\
=1-\frac{(\beta-p) k}{2 \beta} \leqq \frac{p+\beta}{2 \beta} .
\end{gathered}
$$

This contradicts our condition (5.3). Therefore, there is no $z_{0} \in \mathbb{U}$ such that $\left|w\left(z_{0}\right)\right|$ $=1$. This implies that $|w(z)|<1(z \in \mathbb{U})$, that is, that

$$
\left|\frac{\frac{z f^{\prime}(z)}{f(z)}-p}{\frac{z f^{\prime}(z)}{f(z)}+(p-2 \beta)}\right|<1 \quad(z \in \mathbb{U}) .
$$

Thus, we observe that $f(z) \in \mathcal{S}_{p}(0, \beta)$.

Further, we derive

Theorem 5 If $f(z) \in \mathcal{S}_{p}(0, \beta)$ for some real $\beta \geqq p+\frac{1}{2}$, then

$$
\operatorname{Re}\left(\frac{z^{p}}{f(z)}\right)>\frac{1}{2 \beta-2 p+1} \quad(z \in \mathbb{U}) \text {. }
$$

Proof. We consider the function $w(z)$ such that

$$
\frac{z^{p}}{f(z)}=\frac{1+(1-2 \gamma) w(z)}{1-w(z)} \quad(w(z) \neq 1)
$$

for $\gamma=\frac{1}{2 \beta-2 p+1}$ and for $f(z) \in \mathcal{S}_{p}(0, \beta)$.

Then, we know that

$$
\operatorname{Re}\left(\frac{z f^{\prime}(z)}{f(z)}\right)=\operatorname{Re}\left(p-\frac{(1-2 \gamma) z w^{\prime}(z)}{1+(1-2 \gamma) w(z)}-\frac{z w^{\prime}(z)}{1-w(z)}\right)<\beta
$$

for $z \in \mathbb{U}$. 
Since $w(z)$ is analytic in $\mathbb{U}$ and $w(0)=0$, we suppose that there exists a point $z_{0} \in \mathbb{U}$ such that

$$
\max _{|z| \leqq\left|z_{0}\right|}|w(z)|=\left|w\left(z_{0}\right)\right|=1
$$

Then, applying Lemma 1 , we can write that $w\left(z_{0}\right)=\mathrm{e}^{i \theta}$ and $z_{0} w^{\prime}\left(z_{0}\right)=k \mathrm{e}^{i \theta}(k \geqq 1)$. This gives us that

$$
\begin{aligned}
\operatorname{Re}\left(\frac{z_{0} f^{\prime}\left(z_{0}\right)}{f\left(z_{0}\right)}\right)= & \operatorname{Re}\left(p-\frac{(1-2 \gamma) k e^{i \theta}}{1+(1-2 \gamma) \mathrm{e}^{i \theta}}-\frac{k \mathrm{e}^{i \theta}}{1-\mathrm{e}^{i \theta}}\right) \\
= & p+\frac{(1-2 \gamma) k}{2 \gamma}+\frac{k}{2} \\
& \geqq p+\frac{1-\gamma}{2 \gamma}=\beta,
\end{aligned}
$$

which contradicts the inequality (5.10). Thus, there is no point $z_{0} \in \mathbb{U}$ such that $\mid w$ $\left(z_{0}\right) \mid=1$. This means that $|w(z)|<1(z \in \mathbb{U})$, and that,

$$
\operatorname{Re}\left(\frac{z^{p}}{f(z)}\right)>\frac{1}{2 \beta-2 p+1} \quad(z \in \mathbb{U}) .
$$

This completes the proof of the theorem.

Letting $\frac{z f^{\prime}(z)}{p}$ instead of $f(z)$ in Theorem 5 , we have

Corollary 6 If $f(z) \in \mathcal{C}_{p}(\alpha, \beta)$ for some $\beta \geqq p+\frac{1}{2}$, Then

$$
\operatorname{Re}\left(\frac{p z^{p-1}}{f^{\prime}(z)}\right)>\frac{1}{2 \beta-2 p+1} \quad(z \in \mathbb{U}) .
$$

Finally, we consider the coefficient estimates for functions $f(z)$ to be in the classes $\mathcal{S}_{p}(\alpha, \beta)$ and $\mathcal{C}_{p}(\alpha, \beta)$.

Theorem 6 If $f(z) \in \mathcal{A}_{p}$ satisfies

$$
\sum_{n=p+1}^{\infty}\left(\left|n e^{i \alpha}-k\right|+\left|n e^{i \alpha}-(2 \beta-k)\right|\right)\left|a_{n}\right| \leqq\left|p e^{i \alpha}-(2 \beta-k)\right|-\left|p e^{i \alpha}-k\right|
$$

for some real $\alpha\left(|\alpha|<\frac{\pi}{2}\right), \beta(\beta>p \cos \alpha)$, and $k(0 \leqq k \leqq p \cos \alpha)$, then $f(z) \in \mathcal{S}_{p}(\alpha, \beta)$

Proof. It is to be noted that if $f(z) \in \mathcal{A}_{p}$ satisfies

$$
\left|\frac{e^{i \alpha} \frac{z f^{\prime}(z)}{f(z)}-k}{e^{i \alpha \frac{z f^{\prime}(z)}{f(z)}-(2 \beta-k)}}\right|<1 \quad(z \in \mathbb{U})
$$

Then $f(z) \in \mathcal{S}_{p}(\alpha, \beta)$. It follows that

$$
\begin{aligned}
& \left|\frac{\mathrm{e}^{i \alpha} \frac{z f^{\prime}(z)}{f(z)}-k}{\mathrm{e}^{i \alpha} \frac{z f^{\prime}(z)}{f(z)}-(2 \beta-k)}\right|=\left|\frac{\mathrm{e}^{i \alpha} z f^{\prime}(z)-k f(z)}{\mathrm{e}^{i \alpha}-(2 \beta-k) f(z)}\right| \\
& <\frac{\left|p \mathrm{e}^{i \alpha}-k\right|+\sum_{n=p+1}^{\infty}\left|n \mathrm{e}^{i \alpha}-k\right|\left|a_{n}\right|}{\left|p \mathrm{e}^{i \alpha}-(2 \beta-k)\right|-\sum_{n=p+1}^{\infty}\left|n \mathrm{e}^{i \alpha}-(2 \beta-k)\right|\left|a_{n}\right|} .
\end{aligned}
$$


Therefore, if $f(z)$ satisfies the coefficient estimate (5.13), then we know that $f(z)$ satisfies the inequality (5.14). This completes the proof of the theorem.

Letting $\alpha=0$ and $k=p$ in Theorem 6, we have

Corollary 7 If $f(z) \in \mathcal{A}_{p}$ satisfies

$$
\sum_{n=p+1}^{\infty}(n-\beta)\left|a_{n}\right| \leqq(\beta-p)
$$

for some real $\beta\left(p<\beta<p+\frac{1}{2}\right)$, then $f(z) \in \mathcal{S}_{p}(0, \beta)$.

Further, we have

Theorem 7 If $f(z) \in \mathcal{A}_{p}$ satisfies

$$
\sum_{n=p+1}^{\infty} n\left(\left|n e^{i \alpha}-k\right|+\left|n e^{i \alpha}-(2 \beta-k)\right|\right)\left|a_{n}\right| \leqq p\left(\left|p e^{i \alpha}-(2 \beta-k)\right|-\left|p e^{i \alpha}-k\right|\right)
$$

for some real $\alpha\left(|\alpha|<\frac{\pi}{2}\right), \beta(\beta>p \cos \alpha)$ and $k(0 \leqq k \leqq p \cos \alpha)$, then $f(z) \in \mathcal{C}_{p}(\alpha, \beta)$

Corollary 8 If $f(z) \in \mathcal{A}_{p \text { satisfies }}$

$$
\sum_{n=p+1}^{\infty} n(n-\beta)\left|a_{n}\right| \leqq p(\beta-p)
$$

for some real $\beta\left(p<\beta<p+\frac{1}{2}\right)$, then $f(z) \in \mathcal{C}_{p}(\alpha, \beta)$.

\section{Acknowledgements}

This paper was completed when the first author was visiting Department of Mathematics, Kinki University, HigashiOsaka, Osaka 577-8502, Japan, between February 17 and February 26, 2011.

\section{Author details}

${ }^{1}$ Department of Mathematics, Kazim Karabekir Faculty of Education, Ataturk University, Erzuram 25240, Turkey

${ }^{2}$ Department of Mathematics, Kinki University, Higashi-Osaka, 577-8502 Osaka, Japan ${ }^{3}$ Department of Mathematics and Computer Sciences, Faculty of Science and Letters, Istanbul Kultur University, Istanbul, Turkey

Received: 10 April 2011 Accepted: 18 September 2011 Published: 18 September 2011

\section{References}

1. Polatoglu, Y, Blocal, $M$, Sen, A, Yavuz, E: An investigation on a subclass of $p$-valently starlike functions in the unit disc. Turk J Math. 31, 221-228 (2007)

2. Owa, S, Nishiwaki, J: Coefficient estimates for certain classes of analytic functions. J Inequal Pure Appl Math. 3, 1-5 (2002)

3. Aouf, MK, Al-Oboudi, FM, Haidan, MM: On some results for $\lambda$-spirallike and $\lambda$-Robertson functions of complex order. Publ Inst Math. 75, 93-98 (2005)

4. Robertson, MS: Univalent functions $f(z)$ for which $z f^{\prime}(z)$ is spirallike. Michigan Math J. 16, 315-324 (1969)

5. Duren, PL: Univalent Functions. Springer, New York (1983)

6. Miller, SS, Mocanu, PT: Second order differential inequalities in the complex plane. J Math Anal Appl. 65, 289-305 (1978). doi:10.1016/0022-247X(78)90181-6

7. Jack, IS: Functions starlike and convex of order a. J Lond Math Soc. 3, 469-474 (1971). doi:10.1112/j/ms/s2-3.3.469

doi:10.1186/1029-242X-2011-61

Cite this article as: Uyanik et al:: Reciprocal classes of $p$-valently spirallike and $p$-valently Robertson functions. Journal of Inequalities and Applications 2011 2011:61. 